\title{
FERRAMENTA PARA TOMADA DE DECISÃO VISANDO AUMENTO DA EFICIÊNCIA EM REATOR DE EVA COM MINIMIZAÇÃ̃O DE SUBPRODUTOS
}

\author{
L. P. S. ROSA ${ }^{1}$, A. P. PEIXOTO ${ }^{1}$, G. M. N. $\operatorname{COSTA}^{1}$, K. V. PONTES ${ }^{1}$ \\ ${ }^{1}$ Universidade Federal da Bahia, Programa de pós graduação em Engenharia Industrial \\ E-mail para contato: liciannepimentel@hotmail.com
}

\begin{abstract}
RESUMO - O objetivo desde trabalho é propor uma ferramenta para a tomada de decisão aplicada à produção do etileno vinil acetato (EVA) visando minimizar a geração de ácido acético por degradação térmica do polímero na válvula de descarga do reator. Para tanto modelou-se o efeito Joule Thomson visando obter isentálpicas que fornecem a temperatura de estrangulamento versus pressão de saída na válvula. Conhecendo-se do histórico de dados operacionais a relação entre geração de ácido e temperatura, foi possível correlacionar a taxa de geração de ácido em função da pressão de saída na válvula sob diferentes condições operacionais. $\mathrm{O}$ modelo desenvolvido representa uma importante contribuição técnica à planta pois é bastante útil para tomada de decisão, permitindo definir, dentre outras coisas, se a corrente de saída da reação pode ou não ser reciclada, além de possibilitar estudos de otimização, se acoplado a um modelo dos separadores.
\end{abstract}

\section{INTRODUÇÃO}

A reação de copolimerização do eteno com AV (acetato de vinila) para formação do EVA (Etileno Acetato de Vinila) ocorre a altíssimas pressões, cerca de 1.200 bar. A descarga do reator é despressurizada através de uma válvula redutora de pressão para aproximadamente 250 bar a fim de permitir a posterior separação do produto no separador de alta pressão. A corrente que escoa na válvula contém, além do polímero formado, o eteno e o acetato de vinila não reagidos. Tendo em vista a brusca redução de pressão através da válvula, observa-se na prática industrial um aumento de temperatura, devido ao efeito Joule Thomson, que favorece a degradação térmica do EVA a ácido acético. Ao reciclar os reagentes, o ácido pode provocar pontos de corrosão nas linhas e equipamentos da planta, além de provocar pontos de explosão quando em contato com o catalisador. Para não comprometer a segurança do processo, os reagentes remanescentes contaminados pelo ácido são descartados como resíduos se a concentração do ácido formado for alta. Desta forma, o monitoramento da quantidade de ácido formado na válvula de descarga do reator representa uma potencial contribuição para a segurança e economia do processo. Como pode ser constatado, este problema se reveste de importância industrial e necessita de uma abordagem cuidadosa das variáveis envolvidas.

No levantamento bibliográfico realizado a respeito da degradação do EVA, nota-se que os 


\section{9 a 22 de outubro de 2014 \\ Florianópolis/SC}

trabalhos se concentram no estudo das modificações químicas decorrentes da pirólise, a maioria deles utilizando a técnica de análise termogravimétrica (Marcilla et al., 2005; Rimez et al., 2008.). O trabalho de Ovejero et al. (1999) preocupou-se no estudo do tratamento da mistura polimérica contendo o ácido formado. Desta forma, até o conhecimento dos autores, não há na literatura aberta nenhum trabalho que proponha investigar estratégias operacionais para a minimização desse subproduto.

Nesse contexto, a proposta desse trabalho é desenvolver um modelo rigoroso para o processo de estrangulamento na válvula, capaz de prever a quantidade de ácido gerado para uma dada condição operacional, tendo como base os princípios da termodinâmica aplicada a sistemas poliméricos. $\mathrm{O}$ sistema modelado foi EVA/etileno/acetato de vinila. Para tanto, foi desenvolvido inicialmente um modelo para cálculo das isentálpicas que correlacionam a temperatura a jusante da válvula com a pressão de saída a partir do cálculo do efeito Joule Thomsom. A quantidade de ácido gerado é calculada a partir de dados industriais que correlacionam esta variável com a temperatura na saída da válvula. Desta forma, no cenário industrial, a partir de medições de variáveis de processo como temperatura e pressão, é possível estimar, com o modelo aqui desenvolvido, a quantidade de ácido formado e então determinar se a corrente na saída do reator pode ser reciclada ou deve ser descartada. Portanto, o modelo proposto pode auxiliar a tomada de decisão por engenheiros e operadores na planta. Este trabalho representa grande contribuição tanto acadêmica como prática, pois, através da modelagem termodinâmica, é possível mapear as condições de operação que minimizem a formação de ácido, aumentando a rentabilidade e segurança do processo, além de reduzir o impacto ambiental.

Inicialmente, esse artigo apresenta a descrição do processo onde ocorre a reação de degradação térmica do EVA. Posteriormente, é apresentada a abordagem para modelagem desta degradação a partir do cálculo da temperatura de estrangulamento. Em seguida, são discutidos resultados, como as curvas isentálpicas e a sua correlação com a quantidade de ácido gerado.

\section{DESCRIÇÃO DO PROCESSO}

A formação do EVA a partir da copolimerização do eteno com acetato de vinila ocorre a altíssimas pressões, cerca de 1.200 bar. Para permitir a separação dos produtos, a descarga do reator, contendo polímero, etileno e acetato de vinila, é despressurizada através de uma válvula redutora de pressão para cerca 250 bar, como pode ser observado na Figura 1, que é uma seção do fluxograma que representa a produção de EVA e Polietileno de baixa densidade a altas pressões (Folie, 1996).

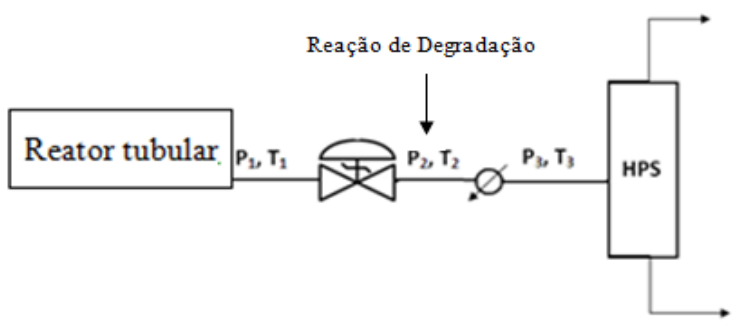

Figura 1- Fluxograma simplificado do estrangulamento na produção de EVA (Folie, 1996). 
Esta brusca queda de pressão em um processo de estrangulamento isentálpico é acompanhada por um aumento de temperatura, conforme observação experimental, devido ao efeito Joule Thomson. Esse aumento de temperatura faz com que o polímero se degrade, formando ácido acético É importante ressaltar que a reação de degradação do EVA cessa durante passagem pelo trocador de calor onde ocorre resfriamento da corrente. Entretanto, a modelagem do trocador está fora do escopo deste trabalho, que se concentra no processo de estrangulamento na válvula. Desta forma, para escopo dessa investigação, considerou-se a degradação que ocorre na tubulação logo após a válvula de descarga do reator de polimerização do EVA, como indicado na Figura 1.

Para o processo investigado, foram coletados em documentos de operação a relação entre a geração de ácido e a temperatura de estrangulamento conforme apresentado na Figura 2. Por motivos de confidencialidade industrial, os valores no gráfico estão normalizados. Observa-se que, à medida que se aumenta a temperatura, ocorre um significativo aumento taxa de produção de ácido, evidenciando que a temperatura de degradação do EVA a ácido acético foi atingida.

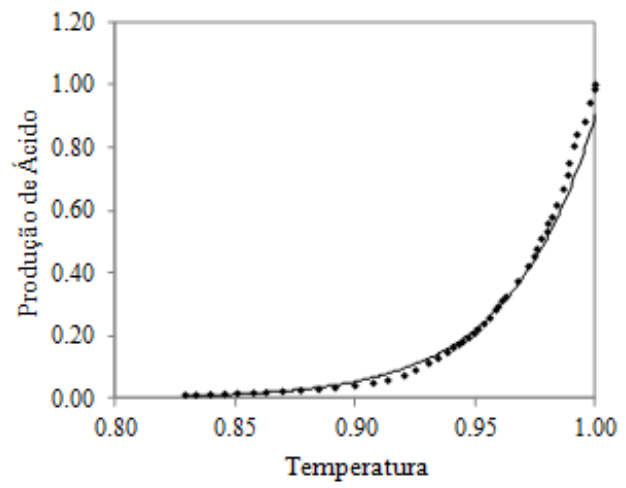

Figura 2 - Geração de ácido acético em função da temperatura.

Em 1989 a empresa japonesa Sulmitono, de consultoria em engenharia, realizou um estudo na planta objeto de estudo desse artigo e forneceu a seguinte relação da taxa de geração de ácido acético com a temperatura a jusante da válvula:

$G_{A A}^{*}=A \cdot \exp \left(-\frac{E}{R T}\right) \cdot P \cdot A V(\%)$

onde $G_{A A}$ é a taxa de geração de ácido acético, $P$, a vazão mássica da corrente, $A V(\%)$ a porcentagem de acetato de vinila incorporado ao EVA e o termo $A \cdot \exp (-E / R T)$ representa a constante cinética. Sendo assim, de posse dos dados apresentados na Figura 3 e da relação funcional da Equação 1, os parâmetros $A$ e $E$ da equação de Arrehnius foram estimados. Obteve-se $A=2,2710^{-13} \mathrm{e}$ $E=48600 \mathrm{~J} / \mathrm{mol} . K$.

A utilização do gráfico da Figura 2 permite uma visualização direta da geração de ácido acético em função da temperatura a jusante da válvula de descarga do reator, sendo portanto a estratégia 
utilizada para a condução deste trabalho. Tendo isso em vista, faz-se necessário calcular a temperatura de estrangulamento em função da pressão de saída na válvula para o sistema polimérico EVA/etileno/acetato de vinila. O modelo proposto correlacionará, portanto, a quantidade de ácido gerado em função da pressão de saída na válvula, permitindo mapear as condições de operação que minimizem a quantidade de ácido acético formado.

É importante ressaltar que, apesar de a indústria já possuir uma relação entre a concentração de ácido e a temperatura do processo, o cálculo desta temperatura, a uma dada condição de processo, não é uma tarefa trivial pois exige um modelo detalhado do processo de estrangulamento na válvula de descarga. Ressalta-se que a modelagem do efeito Joule Thomson para sistemas poliméricos não é uma tarefa trivial e ainda é pouco estudado na literatura, tendo sido foco de trabalhos anteriores do grupo (ROSA et al., 2013a; ROSA et al., 2013b).

\subsection{Modelagem do efeito Joule Thomson para o sistema EVA/AV/etileno}

Na prática, o efeito Joule Thomson é monitorado permitindo-se que o fluido se expanda através de um dispositivo de estrangulamento, que deve estar muito bem isolado para impedir qualquer transferência de calor para ou pelo gás. A taxa de variação da temperatura $T$ em relação à pressão $P$, à entalpia constante $H$, é o coeficiente de Joule-Thomson, $\mu$, definido por:

$\mu=\left(\frac{\partial T}{\partial P}\right)_{H}$

O processo de estrangulamento ocorre ao longo de uma curva à entalpia constante, no sentido de diminuiçaõ da pressão, o que significa que o processo ocorre da esquerda para a direita em um diagrama T-P como ilustrado na Figura 3. À medida que se avança ao longo de uma curva de entalpia constante, a altas pressões, a temperatura aumenta, até a temperatura de inversão. A partir deste ponto, ou seja, para pressões de saída menores que a pressão de inversão, a tempeatura diminui, logo o fluido se resfria. A curva de inversão é a representação geométrica dos pontos de inversão para diferentes condições a montante do estrangulamento.

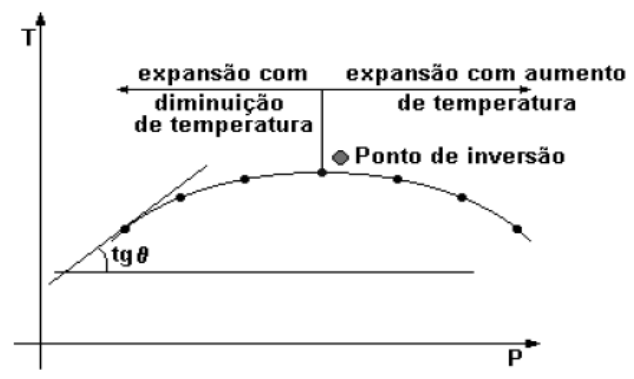

Figura 3- Isentálpica em diagrama TP (Leal, 2012).

A prática usual na literatura é calcular o coeficiente Joule Thomson ou a curva de inversão para avaliar a eficiência de uma equação de estado na representação do processo de 


\section{9 a 22 de outubro de 2014 \\ Florianópolis/SC}

estrangulamento e conhecer apenas se o fluido aqueceu ou resfriou, sem qualquer informação sobre o valor da temperatura de estrangulamento. A metodologia aqui utilizada calcula a temperatura de estrangulamento a partir do conceito de entalpia residual, conforme estudos anteriores do grupo sugerem (Leal, 2012). Pode-se demonstrar (Leal, 2012) que a variação de entalpia no processo de estrangulamento é dada por:

$h^{\text {sistema }}=\left(\Delta h_{T 1 . p 1}^{\prime}\right)+C p^{i d}\left(T_{2}-T_{1}\right)-\left(\Delta h_{T 2 . P 2}^{\prime}\right)$

onde os termos $\left(\Delta h_{T 1, p 1}^{\prime}\right)$ e $\left(\Delta h_{T 2, p 2}^{\prime}\right)$ correspondem à entalpia residual calculadas nas condições antes e depois do estrangulamento, respectivamente, o termo $C p^{i d}\left(T_{2}-T_{1}\right)$, designa a variação

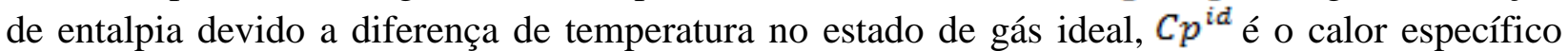
ideal. A equação de estado utilizada para o cálculo da entalpia residual foi a equação PC-SAFT (Perturbed Chain-Statistical Associating Fluid Theory), bastante recomendada na literatura para sistemas poliméricos (Tumakaka et al., 2002; Costa et al., 2010; e Arce et al., 2010 e Sadowski, 2011). Como o processo de estrangulamento ocorre à entalpia constante, a Equação 3 se resume a:

$$
\left(\Delta h_{T 1, p 1}^{\prime}\right)+C p^{i d}\left(T_{2}-T_{1}\right)-\left(\Delta h_{T 2, p 2}^{r}\right)=0
$$

Logo, o problema consiste em encontrar a raiz de uma equação não-linear, no caso a temperatura de estrangulamento $\left(T_{2}\right)$, dadas as condições de entrada $\left(T_{1}, P_{1}\right)$ e a pressão de saída $\left(P_{2}\right)$, além dos parâmetros da equação de estado. $\mathrm{O}$ modelo foi validado em trabalho anterior (ROSA et al., 2013) com dados de literatura e dados industriais da planta de produção de polietileno de alta pressão (PE3) da Braskem, empresa situada no Pólo Petroquímico de Camaçari. Os resultados da temperatura de saída da válvula obtidos nas simulações, quando comparados com os dados industriais, apresentaram um desvio médio de $2.22 \%$. Sendo assim, o modelo para o cálculo da temperatura de estrangulamento para copolímeros está validado.

O desenvolvimento detalhado do modelo, bem como a sua validação, foi publicado em trabalhos anteriores, logo o leitor interessado deve consultá-los (ROSA et al. 2013a e ROSA et al. 2013b). É importante ressaltar que a aplicação desta metodologia para sistemas poliméricos é uma grande contribuição para literatura pois, até conhecimento dos autores, não se encontra a avaliação do efeito Joule Thomson para polímeros.

\section{RESULTADOS}

A partir do modelo validado para cálculo de temperatura de estrangulamento, realizou-se diferentes simulações a fim de obter as isentálpicas para o sistema EVA/AV/etileno nas composições listadas na Tabela 1. Tomou-se como base uma resina comercial, a HM2528, com composição e peso molecular característicos. As isentálpicas foram então obtidas a partir da temperatura de estrangulamento $\left(\mathrm{T}_{2}\right)$ calculada para diferentes pressões na saída da válvula $\left(\mathrm{P}_{2}\right)$, considerando uma condição de alimentação fixa, determinada por recomendação industrial. 
Tabela 1- Composições do sistema EVA/etileno/acetato de vinila ${ }^{[1]}$.

\begin{tabular}{|c|l|l|l|}
\hline Componente & Composição 1 (X1) & Composição 2 (X2) & Composição 3 (X3) \\
\hline EVA & 0.38 & 0.4 & 0.6 \\
\hline Etileno & 0.37 & 0.4 & 0.25 \\
\hline AV & 0.25 & 0.2 & 0.15 \\
\hline
\end{tabular}

A Figura 4 ilustra as três isentálpicas para as diferentes composições. Por se tratar de condições de saída calculadas a partir de uma dada condição de entrada, as curvas não partem do mesmo ponto em $P=1250$ atm. Nota-se que a composição da mistura polimérica e as condições de operação a montante da válvula de descarga são fatores determinantes na temperatura de estrangulamento. Percebe-se que, à medida que o teor de EVA aumenta, a temperatura de estrangulamento diminui, uma importante observação é a ausência da temperatura de inversão, uma característica bastante particular apresentada por poucos fluidos, como o hidrogênio. Outra observação da Figura 4 é a influência das condições de pressão e temperatura na entrada sobre a temperatura de estrangulamento - essa análise pode ser feita ao deslocar-se ao longo das isentálpicas a partir da condição de alimentação.

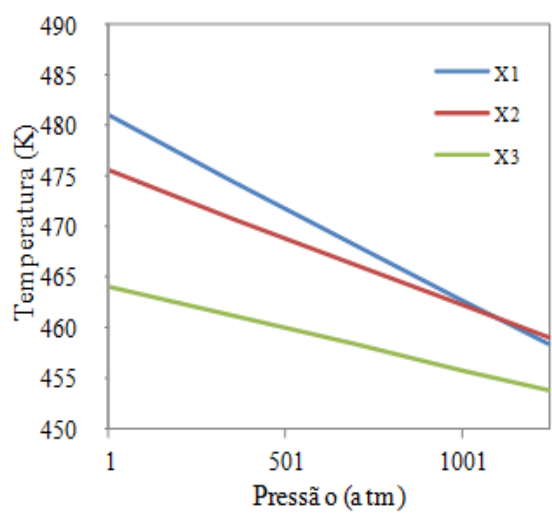

Figura 4 -Isentálpicas para o sistema EVA/AV/etileno com as composições: (X1), (X2) e (X3).

Uma vez conhecendo-se a temperatura de estrangulamento para diferentes pressões de saída, ou seja, as isentálpicas, calculou-se a quantidade de ácido acético a partir da Equação 1. Para essa investigação, utilizou-se as diferentes composições listadas na Tabela 1 e os resultados estão ilustrados na Figura 5. 


\section{9 a 22 de outubro de 2014 \\ Florianópolis/SC}

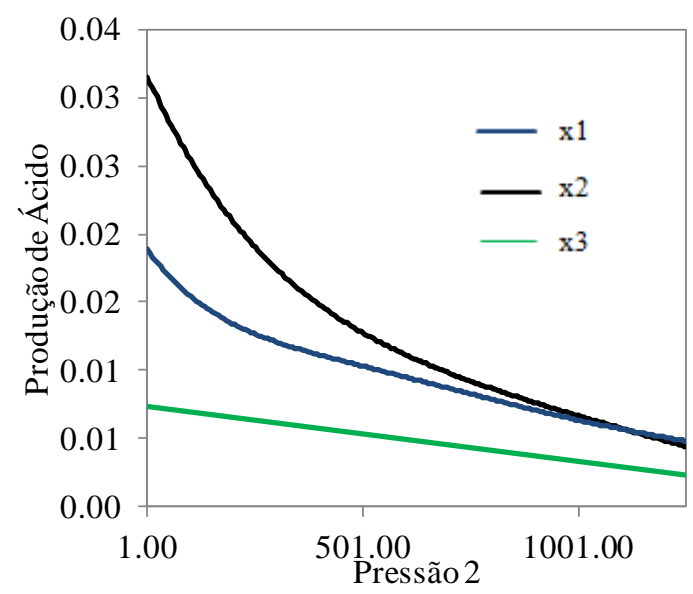

Figura 5 - Geração do ácido acético versus pressão de saída da válvula.

Nota-se que, quanto maior a queda de pressão na válvula, maior a temperatura de estrangulamento, e portanto mais ácido é formado. Este é um resultado quantitativo importante porém não suficiente para a determinação das condições ótimas de operação, já que deve ser levada em conta restrições operacionais e a eficiência dos equipamentos a jusante da válvula: o trocador de calor e o vaso de separação (Figura 1). Percebe-se também que, quanto maior a quantidade de acetato de vinila maior a geração de ácido. Este fato confirma a previsão da literatura (Marcilla et al., 2005; Rimez et al., 2008;). $\quad$ Ressalta-se que o modelo apresentado permite calcular a quantidade de ácido gerado para diferentes condições operacionais, descritas por temperatura e pressão, além de informações da resina produzida como composição e peso molecular, todas disponíveis no ambiente industrial.

$\mathrm{Na}$ literatura comumente se calcula o valor do coeficiente de Joule Thomson, que permite conhecer apenas se o fluido aqueceu ou resfriou, sem qualquer informação sobre o valor da temperatura de estrangulamento. Sem o conhecimento desta variável, não seria possível correlacionar a pressão com a quantidade de ácido gerado. Desta forma, o cálculo das isentálpicas para diferentes condições de temperatura, pressão e composição é uma importante contribuição deste trabalho, sobretudo pela aplicação a sistemas poliméricos (ROSA et al., 2013a e ROSA et al., 2013b).

\section{CONCLUSÕES}

O modelo desenvolvido permite estimar em tempo real a quantidade de ácido formado, fornecendo assim subsídios confiáveis para tomada de decisão em relação ao reciclo da corrente para o reator. Ele apresenta ainda potencial para otimização do processo se acoplado com o modelo da bateria de separadores já que a eficiência da separação está relacionada com a condição de pressão e temperatura na saída da válvula. Ressalta-se a contribuição deste trabalho para a eficiência e segurança do processo, que pode ser traduzida em ganhos econômicos e ambientais. 


\section{REFERÊNCIAS}

ARCE, P. F.,AZNAR, M. Thermodynamic modeling of liquid-fluid phase equilibrium in supercritical ethylene plus copolymer plus co-solvent systems using the PC-SAFT equation of state. Journal of Supercritical Fluids, 2010.

COSTA, G. M. N., KISLANSKY, S, Guerrieri, Y, PESSOA, F. L. P., MELO, S.A.B.V. EMBIRUCU, M. Calculation of Pressure-Temperature Diagrams and Distance for Phase Transition in Polyethylene Solutions. Ind. \& Engineering Chemistry Research. Vol.49, 2010.

LEAL, D. S. Avaliação de equações de estado no cálculo de propriedades do dióxido de carbono úteis ao estudo do seu escoamento. Mestrado em engenharia industrial. UFBA, 2012.

MARCILLA, A., GOMEZ-SIURANA, A., MENARGUES, S. Qualitative study of the evolution of the composition of the gas evolved in the thermal and HY-catalytic oxidative degradation of EVA copolymers. Thermochimica Acta. Volume: 438, 2005.

OVEJERO, G.; SÁNCHEZ, A.; DURÁN, A. Removal of acetic acid by adsorption from an ethylene recycle stream in the ethylene-vinyl acetate copolymerization process. Separation Science and Technology, v 34, n 3, p 525-543, Feb 1999.

FOLIE, B.; RADOSZ, M., "Phase Equilibria in High-Pressures Polyethylene Technology", and. Eng. Chem. Res., v. 34, p. 1501-1516, (1996).

RIMEZ, B., RAHIER, H., VAN ASSCHE, G., ARTOOS, T., BIESEMANS, M., VAN MELE, B. The Thermal Degradation of Poly(Vinyl Acetate) And Poly(Ethylene-Co-Vinyl Acetate), Part I: Experimental Study of the Degradation Mechanism. Polymer Degradation And Stability. Volume: 93, 2008.

ROSA, L.P.S, PEIXOTO, A. P, PONTES, K. V., GUERRIERI, Y, COSTA, G. M. N. Utilização da equação de estado PC-SAFT para predição de propriedades termodinâmicas para o sistema etileno/PEBD. $12^{\circ}$ Congresso Brasileiro de Polímeros, setembro de $2013 \mathrm{a}$.

ROSA, L.P.S, PONTES, K. V, PEIXOTO, A. P, ., COSTA, G. M. N. Determinação de temperaturas ótimas em processos de estrangulamento ao longo do escoamento. $X X X I I I^{\circ}$ Encontro Nacional de Engenharia de Produção, outubro de 2013 b.

SADOWSKI, G. Modeling of Polymer Phase Equilibria Using Equations of State Polymer Thermodynamics: Liquid Polymer-Containing Mixtures. Advances In Polymer Science. Volume: 238,2011 .

TUMAKAKAA, F, GROSS B, J. SADOWSKIA, G. Modeling of polymer phase equilibria using Perturbed-Chain SAFT. Fluid Phase Equilibria. Volumes 194-197, 30 March 2002, Pages 541551. 